\title{
Guibitang, a traditional herbal medicine, induces apoptotic death in A431 cells by regulating the activities of mitogen-activated protein kinases
}

\author{
Nam-Hui Yim, Aeyung Kim, Chun Liang, Won-Kyung Cho and Jin Yeul Ma*
}

\begin{abstract}
Background: Guibi-tang (GBT), a traditional herbal formula, mainly has been shown to possess immune regulation, antioxidant and protective effect of the gastric mucosa. Constituent herbs of GBT are frequently used to treat various diseases; however, their pharmacological effects, especially on cancer cells, differ from those of GBT. Furthermore, the molecular mechanisms behind effects of GBT remain unclear. In the present study, we explored the mechanism of chemopreventive/chemotherapeutic efficacy of GBT against human squamous cell carcinoma without cytotoxicity in normal cells and proved the efficacy of GBT through performing in vivo xenograft assay.

Methods: For analysis of the constituents of GBT, high performance liquid chromatography (HPLC)-DAD system was performed. To detect the anticancer effect of GBT, cell viability assay, caspase activity assay, cell cycle analysis, DNA fragmentation analysis, and Western blot analysis were performed in A431 cells. In addition, the inhibitory effect of tumor growth by GBT was evaluated in athymic nude mice inoculated with A431 cells.

Results: GBT showed cytotoxic activity against three different squamous cell carcinoma, especially on A431 cells. GBT induced the apoptosis through activating the caspase-8 in A431 cells. Inhibition of A431 cell growth by GBT was caused by G1-phase arrest through regulating proteins associated with cell cycle progression, such as cyclin D1, p21, and p27. Furthermore, GBT regulated the activation of mitogen-activated protein kinases (MAPKs) including extracellular signal-regulated kinase (ERK), p38 and c-Jun NH2-terminal kinase (JNK), and activated p53, a tumor suppressor protein. In MAPKs inhibitor study, inhibitors respectively blocked GBT-induced cell viability, indicating that MAPKs signals play critical role in cell death caused by GBT. In vivo xenografts, daily oral administration of $600 \mathrm{mg} / \mathrm{kg}$ GBT efficiently suppressed the tumorigenic growth of A431 cells without side effects such as loss of body weight and change of toxicological parameters compared to vehicle.
\end{abstract}

Conclusions: We first elucidate that GBT stimulates the apoptotic signaling pathway and suppresses the proliferation of A431 cells via regulating MAPKs signaling pathway. Furthermore, GBT significantly inhibits tumor growth of A431 cells without causing systemic toxicity. Based on our study, GBT could be useful in the management of skin cancer as chemoprevention and chemotherapy remedy.

Keywords: Guibitang (GBT), Squamous carcinoma cells, Anti-cancer effect, Apoptosis, Mitogen-activated protein kinases

\footnotetext{
* Correspondence: jyma@kiom.re.kr

Korean Medicine (KM)-Based Herbal Drug Development Group, Korea

Institute of Oriental Medicine (KIOM), Daejeon 305-811, Korea
} 


\section{Background}

Basal cell carcinoma (BCC) and squamous cell carcinoma (SCC) are commonly referred to as non-melanoma skin cancers $[1,2]$. BCC is a slow-growing cancer that does not usually metastasize. Similarly, SCC is frequently localized without evidence of blood-born metastasis, making direct treatment of the tumor straightforward. However, SCC is the sixth most common cancer worldwide, and its incidence has increased dramatically at multiple sites in the body, including the head and neck, cervix, and lung $[3,4]$. Accordingly, it is necessary to develop novel effective chemopreventive agents to inhibit the development of SCC.

Guibitang (GBT), known as 'Kihi-to' in Japan and 'GuiPi-Tang' in China, is a traditional medicine and herbal formula that has been used for several hundred years, predominantly to treat insomnia, amnesia, palpitations, anxiety, fatigue, poor appetite, and depression [5]. Recent studies have reported the specific bioactivities of GBT, which include immune regulation [6], antioxidant effects [7], and protective effect of the gastric mucosa [8]. GBT is an aqueous polyherbal formulation that contains 12 herbs: Angelica gigas Nakai, Dimocarpus longan, Zizyphus jujuba Miller (seed), Polygala tenuifolia, Panax ginseng, Astragalus membranaceus, Atractylodes ovate, Poria cocos, Inula helenium, Glycyrrhiza glabra, Zingiber officinale, and Zizyphus jujuba Miller (Fructus). GBT also regulates chronic fatigue syndrome-associated cytokine production, whereas the addition of Gardenia jasminoides, Paeonia suffruticosa, and Bupleurum falcatum to GBT improves palliative care in patients undergoing chemotherapy for ovarian cancer [9]. Although it has been shown that adding several herbs to GBT results in anti-cancer effects against gynecological or lung cancer, the molecular mechanisms behind these effect of GBT remain unclear. Tumorigenesis is caused by unregulated growth of cells resulting from DNA damage, mutations of functional genes, dysregulation of the cell cycle, and loss of apoptotic function [10]. Therefore, regulating the induction of apoptosis by modulating cell growth and survival-related signaling pathways is a common and major target for cancer therapies [11]. Among several signaling pathways in cancer cells, mitogen-activated protein kinase (MAPK) signals including extracellular signal-regulated kinases (ERK), p38 kinases, and c-Jun Nterminal kinases (JNK), take an important role in cell death and survival [12]. The regulation of ERK activation is induced by conditions of stress such as some agents and oxidant injury, which plays a major role in regulating cell growth and differentiation [13]. JNK and p38 are activated in response to several stress signals including tumor necrosis factor and hyperosmotic condition, which is associated with induction of apoptosis [14]. In the present study, we evaluated whether GBT shows the anti- cancer effect in A431 human squamous carcinoma cells, which demonstrated that GBT induces apoptosis of cancer cells specifically, as an inhibition of the cell growth via regulating MAPK signaling pathway in A431 cells.

\section{Methods}

\section{Cell culture}

Various human cancer cell lines, obtained from the Korean Cell Line Bank (KCLB, Seoul, Korea) and American Type Culture Collection (ATCC, Rockville, MD), were cultured in Dulbecco's modified Eagle's medium (DMEM) and RPMI-1640 (Lonza, Walkersville, MD) supplemented with $10 \%$ fetal bovine serum (FBS; Hyclone, Logan, UT). Primary hepatic cells obtained from mice were grown in Williams E Medium (GIBCO, Gaithersburg, MD) supplemented with 10\% FBS. All media contained $100 \mathrm{U} / \mathrm{mL}$ penicillin $\mathrm{G}$ and $100 \mu \mathrm{g} / \mathrm{mL}$ streptomycin (GIBCO). Cells were incubated in a humidified $5 \% \mathrm{CO}_{2}$ atmosphere at $37^{\circ} \mathrm{C}$.

\section{Herb materials and preparation of GBT}

GBT was composed of 12 medicinal herbs; their constitution ratio is shown in Table 1 . The 12 herbs were purchased from the Korea Medicine Herbs Association (Yeongcheon, Korea). The herbal mixture was extracted by heating in water of 8-10 fold the herb weight for $3 \mathrm{~h}$ at $115^{\circ} \mathrm{C}$ on Cosmos-600 extractor (Incheon, Korea). After boiling, the extract was filtered out using standard testing sieves (pore size $150 \mu \mathrm{m}$, Retsch, Germany) and prepared in the form of powder by freeze-drying. $50 \mathrm{mg}$ of GBT powder was dissolved in $1 \mathrm{~mL}$ of distilled water, passed through a $0.22 \mu \mathrm{m}$ filter, and stored at $-20^{\circ} \mathrm{C}$ before use.

\section{Table 1 Composition of the Guibitang (GBT) preparation}

\begin{tabular}{llc}
\hline Scientific name & Part used & Amount used (g) \\
\hline Angelica gigas Nakai & Radix & 4 \\
Dimocarpus longan & Fructus & 4 \\
Zizyphus jujuba Miller & Seed & 4 \\
Polygala tenuifolia & Rhizoma & 4 \\
Panax ginseng & Radix & 4 \\
Astragalus membranaceus & Radix & 4 \\
Atractylodes ovata & Rhizoma & 4 \\
Poria cocos & Radix & 4 \\
Zingiber officinale Rosc. & Rhizoma & 2.48 \\
Inula helenium L. & Radix & 2 \\
Zizyphus jujuba Miller & Fructus & 2 \\
Glycyrrhiza glabra Fisch & Radix & 1.2 \\
Total amount & & 44.69 \\
\hline
\end{tabular}




\section{HPLC analysis}

Standardization of herbal extracts was performed by highperformance liquid chromatography (HPLC) fingerprinting with chemical standards purchased from Wako Pure Chemical Industries (Japan; liquiritin), the Korea Food \& Drug Administration (KFDA; 6-gingerol), Elcom Science (Korea; decursinol, decursin, and decursinol angelate), Chengdu Must Bio-Technology (China; onjisaponin B), and Sigma-Aldrich (USA; spinosin, vanilylacetone, nodakenin, nodakenetin, liquiritigenin, ginsenoside Rg1 and $\mathrm{Rb} 1$, calycosin, jujuboside A, formononetin, atractylenolide I, II and III, costunolide, and pachymic acid). Standard solutions were prepared by dissolving each marker component in $100 \%$ methanol at $1 \mathrm{mg} / \mathrm{mL}$. GBT powder was weighed accurately and dissolved in $60 \%$ methanol at $50 \mathrm{mg} / \mathrm{mL}$ for analysis. The HPLC-DAD system used (Hitachi Co., Japan) consisted of a pump (L-2130), autosampler (L-2200), column oven (L-2350), and diode array UV/VIS detector (L-2455). Output signals from the detector were recorded using EZChrom Elite software from Hitachi. For sample analysis, a HECTOR-A-C18 column ( $5 \mu \mathrm{m}, 4.6 \times 250 \mathrm{~mm}$, RS Tech, Korea) was used and the UV wavelengths were 203 and $254 \mathrm{~nm}$. The mobile phase used was water (A) and acetonitrile (B) at a flow rate of $1.0 \mathrm{~mL} / \mathrm{min}$ and the column temperature was maintained at $40^{\circ} \mathrm{C}$. The elution conditions applied during the analysis were as follows: $0-10 \mathrm{~min}$, isocratic $1 \% \mathrm{~B} ; 10-70 \mathrm{~min}$, linear gradient $1-50 \% \mathrm{~B} ; 70-80 \mathrm{~min}$, linear gradient $50-100 \% \mathrm{~B}$; and $80-90 \mathrm{~min}$, isocratic $100 \% \mathrm{~B}$. The sample injection volume was $10 \mu \mathrm{L}$. Figure 1 shows the HPLC fingerprints of GBT extracts (Table 2).

\section{Cell viability assay}

Cells $\left(4 \times 10^{3}\right.$ or $5 \times 10^{3}$ per well) were inoculated in a 96-well plate and treated with GBT for 24 or $48 \mathrm{~h}$. After incubation, cell viability was analyzed by 3-[4, 5dimethylthiazol-2-ly]-2, 5-diphenyl-tetrazolium bromide (MTT) assay as described previously [15]. For growth analysis, cells were seeded at a density of $1 \times 10^{5} / \mathrm{mL}$ and treated with GBT for 1, 2, or 3 days. The cells were counted and the doubling time was calculated using an online tool (www.doubling-time.com/compute.php).

\section{Cell cycle analysis}

Cells were seeded at a density of $1 \times 10^{5} / \mathrm{mL}$ and treated with GBT for 12 or $24 \mathrm{~h}$. The propidium iodide (PI;

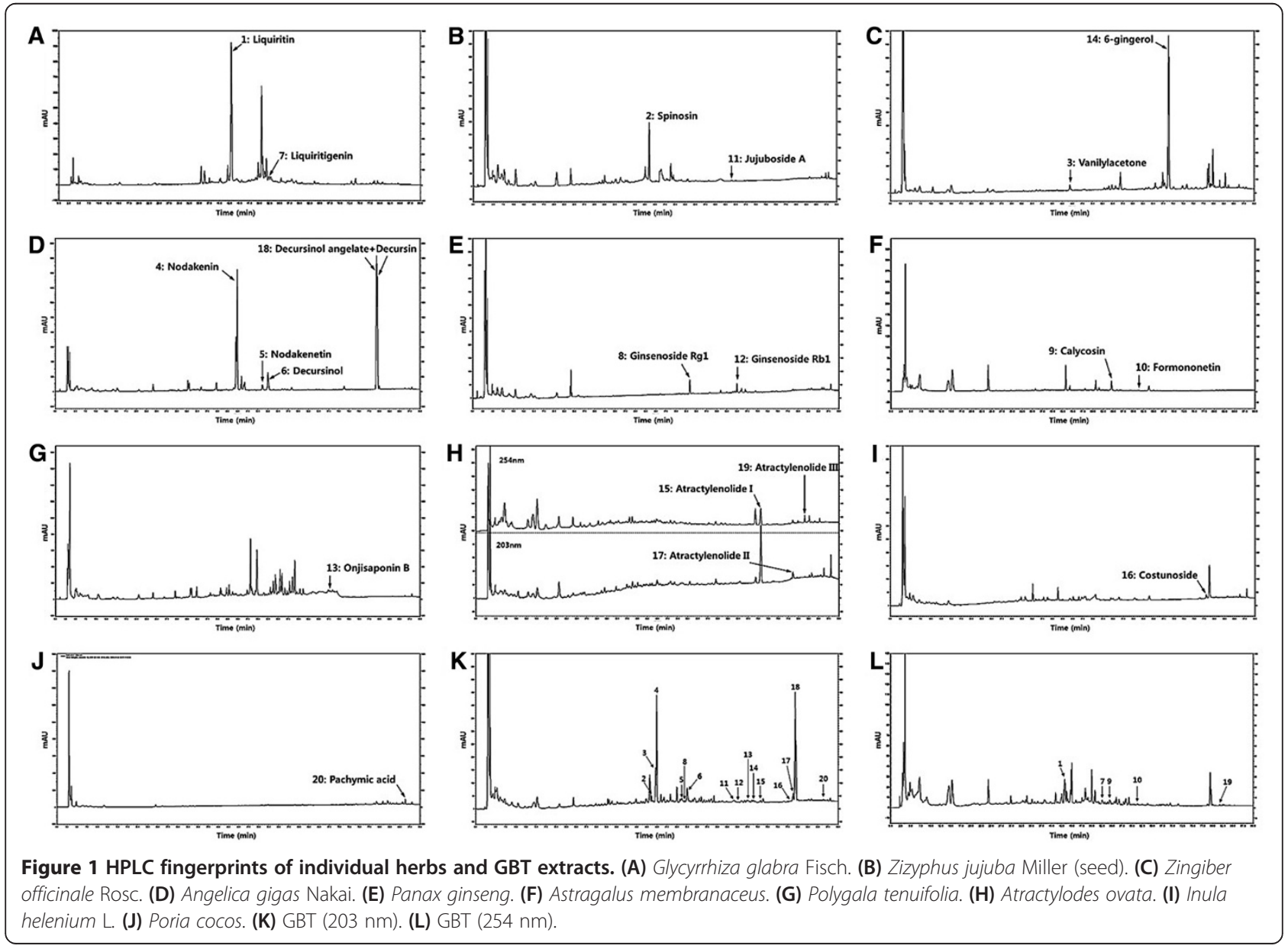


Table 2 Characterization of standard compounds in GBT by HPLC

\begin{tabular}{lllll}
\hline $\begin{array}{l}\text { Peak } \\
\text { no. }\end{array}$ & Compound name & Classification* & tR (min) & $\begin{array}{l}\text { Wavelength } \\
\text { (nm) }\end{array}$ \\
\hline 1 & Liquiritin & 12 & 43.25 & 254 \\
2 & Spinosin & 3 & 43.71 & 203 \\
3 & Vanilylacetone & 9 & 44.70 & 203 \\
4 & Nodakenin & 1 & 44.99 & 203 \\
5 & Nodakenetin & 1 & 51.18 & 203 \\
6 & Decursinol & 1 & 52.55 & 203 \\
7 & Liquiritigenin & 12 & 52.75 & 254 \\
8 & Ginsenoside Rg1 & 5 & 51.37 & 203 \\
9 & Calycosin & 6 & 54.40 & 254 \\
10 & Formononetin & 6 & 61.26 & 254 \\
11 & Jujuboside A & 11 & 64.18 & 203 \\
12 & Ginsenoside Rb1 & 5 & 65.16 & 203 \\
13 & Onjisaponin B & 4 & 67.59 & 203 \\
14 & 6-gingerol & 9 & 69.00 & 203 \\
15 & Atractylenolide I & 7 & 70.75 & 254 \\
16 & Costunolide & 10 & 78.16 & 203 \\
17 & Atractylenolide II & 7 & 78.84 & 203 \\
18 & Decursin + Decursinol & 1 & 79.35 & 203 \\
& angelate & & 81.67 & 254 \\
19 & Atractylenolide III & 7 & 8 & 203 \\
\hline 10 & Pachymic acid & 8.36 & \\
\hline
\end{tabular}

"1, Angelica gigas Nakai; 2, Dimocarpus longan (not detected); 3, Zizyphus jujuba Miller (seed); 4, Polygala tenuifolia; 5, Panax ginseng; 6, Astragalus membranaceus; 7, Atractylodes ovate; 8, Poria cocos; 9, Zingiber officinale Rosc.; 10, Inula helenium L.; 11, Zizyphus jujuba Miller (fructus); 12, Glycyrrhiza glabra Fisch.

Sigma-Aldrich, St. Louis, MO) staining for cell cycle analysis were performed as described previously [15]. DNA contents of the stained cells were analyzed by FACS Calibur flow cytometry using Cell Quest software (Becton-Dickinson, Franklin Lakes, NJ).

\section{Caspase activity assay}

To determine caspase-3/7 activity, cells were seeded at a density of $1 \times 10^{4} /$ well in a 96-well plate and treated with GBT for $24 \mathrm{~h}$. Caspase activity was measured in triplicate by using a Caspase-Glo $3 / 7$ assay kit (Promega, Madison, WI) according to the manufacturer's instructions. Culture medium was used as a blank control and luminescence was measured using an MLX microtiter luminometer (Dynex Technologies Inc., Chantilly, VA).

\section{DNA fragmentation analysis}

To investigate the apoptotic effect of GBT, we assessed oligonucleosomal DNA fragmentation by agarose gel electrophoresis. Cells were harvested at 12 and $24 \mathrm{~h}$ after treatment. Genomic DNA was prepared from harvested cells using a Genomic DNA Purification Kit (Promega, Madison, WI) according to the manufacturer's instructions. It was then subjected to electrophoresis on a $1.5 \%$ agarose gel impregnated with ethidium bromide reagent (Sigma-Aldrich, St. Louis, MO) to detect ladder formation.

\section{Western blot analysis}

The cell lysates treated with GBT for western blot analysis were prepared as described previously [15]. The same amount of protein for each sample was electrophoresed and transferred onto a polyvinylidene difluoride (PVDF) membrane (Millipore, Billerica, MA). Proteins were detected using primary antibodies specific for cyclin D1, cyclin B1, p21, p27, caspase-3, caspase-8, caspase-9, Bid, Bax, Bcl-2, PARP, ERK, phospho-ERK, p38, phospho-p38, JNK, phospho-JNK, p53, GAPDH, and $\beta$-actin, all of which were obtained from Cell Signal Technology. This was followed by incubation with HRP-conjugated secondary antibodies for $1 \mathrm{~h}$ at room temperature. The specific protein was detected using the enhanced chemiluminescence imaging system (CoreBio, Seoul, Korea).

\section{Animals and tumor xenografts}

Female mice (Athymic nu/nu, 8 weeks, 25-29 g; NARA Bio, Seoul, Korea) were acclimated under conditions of constant temperature $\left(24 \pm 1^{\circ} \mathrm{C}\right)$ and humidity $(55 \pm 15 \%)$ with $12 \mathrm{~h}$ light/dark cycle for 1 week. Mice were injected subcutaneously with $3 \times 10^{6}$ A431 cells/100 $\mu \mathrm{L}$ harvested and suspended in DMEM medium without FBS. Mice with palpable tumors were divided into two groups for study, and group 1 (vehicle, $\mathrm{n}=5$ ) mice received the injection of physiological saline (Choongwae Normal Saline Inj., Korea), whereas group 2 (GBT, $\mathrm{n}=5$ ) orally received GBT. The administrated amount of GBT for human adults with an average body weight of $60 \mathrm{Kg}$ is approximately 12 36 g/day and the yield of powdered extraction is approximately $30 \%(\mathrm{wt} / \mathrm{wt})$. Based on this estimation data, GBT at the doses of $600 \mathrm{mg} /$ day $/ \mathrm{Kg}$ of body weight was orally administered to mice for 14 days. GBT treatment was started at day 3 following the tumor cell inoculation. Tumor volume was monitored using electronic caliper on every alternate day and tumor volume was calculated using following formula: tumor volume = length $\times$ width $\times$ width $/ 2$. The experiment was terminated at the end of 15 days when the vehicle-treated animals had large tumors, which was sacrified by obtaining blood from abdominal vein. For determining the toxicity of GBT, chemical analysis of serums obtained from mice was determined using an Auto Biochemistry Analyzer (XL-200, Erba Diagnostics, Mannheim, Germany) and complete blood cell count $(\mathrm{CBC})$ from mice was analyzed using a ADVIA 2120i Hematology System (Siemens Healthcare, Camberley, UK). The animal experimental 
procedures were approved by Korea Institute of Oriental Medicine Care and Use Committee with a reference number of \#12-094 and \#13-030, and performed in accordance with the Korea Institute of Oriental Medicine Care Committee Guide lines.

\section{Statistical analysis}

Data are presented as means \pm SD. Student's $t$-test was employed to assess the statistical significance of differences between the control and GBT-treated groups. Values of $p<0.05$ and $<0.01$ were considered to indicate statistical significance.

\section{Results}

\section{GBT decreases cell viability in A431 human squamous carcinoma cells}

Six different human cancer cell lines (A431 [squamous], AGS [stomach], HeLa [cervical], Caki-1 [kidney], SKHep-1 [liver], and HCT116 [colon]) were treated with $500 \mu \mathrm{g} / \mathrm{mL}$ GBT for $48 \mathrm{~h}$, and cell viability was assessed by an MTT assay. Although most cell lines were unaffected, the viability of A431 cells was inhibited $>35 \%$ by treatment with GBT (Figure 2A). Therefore, subsequent tests focused on A431 cells. To further define the inhibitory action of GBT on SCCs, the suppression of cell growth by GBT on three different SCC lines (SCC12, SCC13, and A431) was evaluated. As shown in Figure 2B, treatment with 500 and $1000 \mu \mathrm{g} / \mathrm{mL}$ GBT for 48 h reduced the viability of A 431 cells by $35 \%$ and $52 \%$, respectively. Treatment of SCC13 cells with $1000 \mu \mathrm{g} / \mathrm{mL}$ GBT also inhibited the cell growth by $~ 30 \%$ although these effects were not as potent as those observed in A431 cells. In contrast, the viability of SCC12 cells was not affected significantly by GBT. The potential cytotoxic effect of GBT on normal cells was assessed using normal human $\mathrm{HaCaT}$ keratinocytes and mouse primary liver cells. HaCaT cells were unaffected by GBT under the same conditions that were cytotoxic to A431 cells (Figure 2C). In addition, no cytotoxic effects on primary liver cells were observed by treatment with $500 \mu \mathrm{g} / \mathrm{mL}$ or $1000 \mu \mathrm{g} / \mathrm{mL}$ GBT. Instead, GBT weakly increased the viability of liver primary cells in a dose- and timedependent manner (Figure 2D). These results suggest that GBT has cancer-specific cytotoxic effect on A431 cells, without affecting normal cells.

GBT causes cell cycle arrest in G1 and increases the subG1 population in $\mathrm{A} 431$ cells

A431 cells were treated with 500 and $1000 \mu \mathrm{g} / \mathrm{mL}$ GBT for 12 or $24 \mathrm{~h}$, and cell cycle progression was assessed. Cells were stained with PI, and the percentage of cells at each stage of the cell cycle was quantified using flow cytometry. As shown in Figure 3A, GBT increased the number of cells in the sub-G1 peak in a time- and dosedependent manner. After $24 \mathrm{~h}$ treatment with 500 and $1000 \mu \mathrm{g} / \mathrm{mL}$ GBT, $8.64 \%$ and $9.27 \%$ of cells had accumulated in the sub-G1 phase, respectively, which represented up to a nine-fold increase compared to untreated
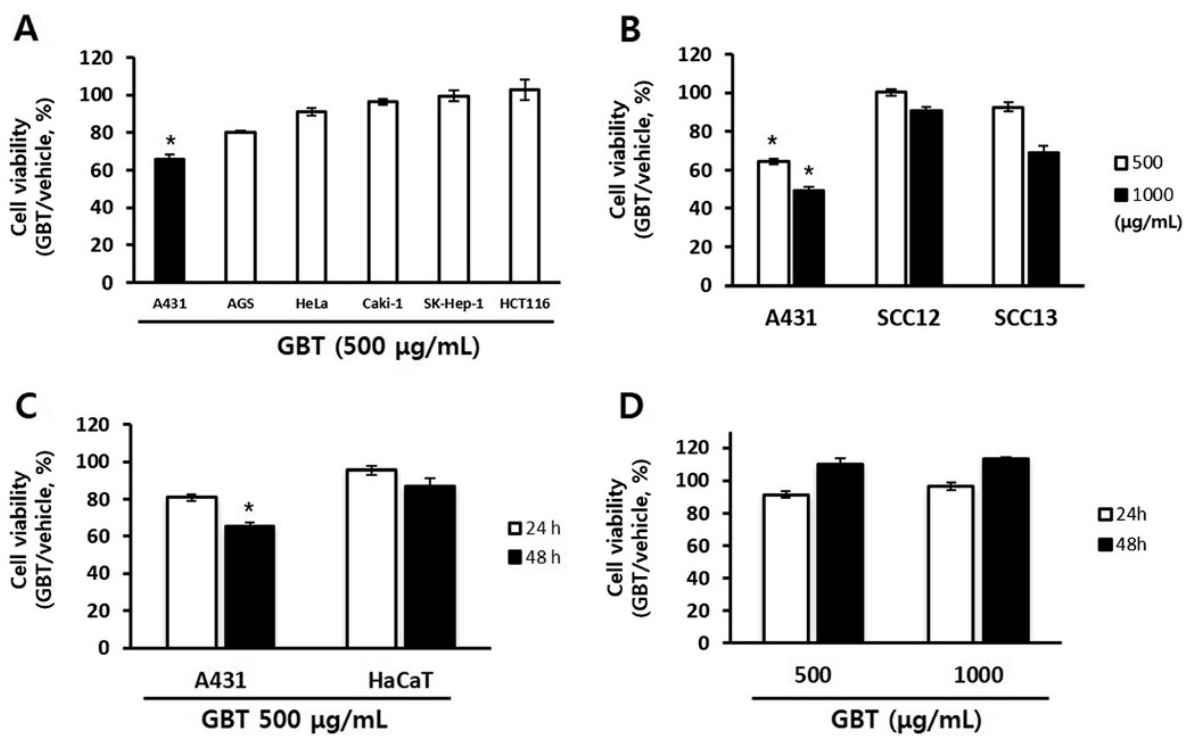

Figure 2 Anti-proliferative effect of GBT on human cancer cells. Cell viability was determined by MTT assay. The results are expressed as percentages of viable cells compared to untreated cells (vehicle). Data show the means \pm SD of two independent triplicate experiments. (A) Inhibition of cell viability by GBT in several human cancer cell lines for $48 \mathrm{~h}$. (B) Comparison of the effects of GBT on the viability of three squamous carcinoma cell lines: A431, SCC12, and SCC13 for 48 h. (C) Effect of GBT on the growth of A431 and HaCaT cells for 24 and 48 h, respectively. (D) Comparison of GBT-induced cytotoxicity between A431 cells and mouse primary liver cells (as a normal cells) in a dose- and time-dependent manner. 




cells (CTL). In addition, $56.09 \%$ and $52.75 \%$ of cells treated with 500 and $1000 \mu \mathrm{g} / \mathrm{mL}$ GBT accumulated in G1 after $12 \mathrm{~h}$, and remained comparable after $24 \mathrm{~h}$. Under the same conditions, the accumulation of cells in $\mathrm{S}$ and G2/M phases were decreased by GBT compared to CTL.

Based on these data, we investigated whether the expressions of cell cycle-regulating proteins are affected by treatment with GBT. As shown in Figure 3B, treatment with GBT altered the expression of proteins associated with G1 phase progression. Specifically, the expression of p21 and p27 was increased, whereas cyclin D1 levels were reduced. In contrast, levels of cyclin B1, which regulates the G2/M phase, was unaffected by GBT. The results shown in Figure $3 \mathrm{C}$ confirm that the antiproliferative effects of GBT are due to the induction of G1 arrest. Proliferation of A431 cells was inhibited after 24 h treatment with GBT, and the number of cells was reduced two-fold by GBT compared to controls after $72 \mathrm{~h}$. These data indicate that induction of G1 cell cycle arrest by GBT hinders cell growth, which is related to cell death in A431 cells.
GBT-stimulated activation of pro-apoptotic proteins and DNA fragmentation is attributable to the induction of apoptosis in A431 cells

To assess whether GBT-induced cytotoxicity may be related to apoptosis, we assessed DNA fragmentation using gel electrophoresis (Figure 4A). An increase in the amount of fragmented oligonucleosomal-length DNA was detected after $24 \mathrm{~h}$ treatment with $500 \mu \mathrm{g} / \mathrm{mL}$ GBT, but not at $12 \mathrm{~h}$. To confirm these observations, the activation of caspase-3/-7, a key apoptotic mediator, was analyzed in A431 cells treated with GBT for $24 \mathrm{~h}$. As expected, GBT increased caspase-3/-7 activity significantly in a dose-dependent manner (Figure 4B). Next, Western blotting revealed that procaspase- 3 and -8 , but not -9 , were cleaved to their active form after exposure to GBT. Furthermore, GBT induced the cleavage of PARP, a substrate of active caspase- 3 , in a dosedependent manner. In contrast, no Bid truncation was detected and the expression of Bax was unaffected by GBT despite the decrease of Bcl-2 levels (Figure 4C). To confirm that the apoptosis induced by GBT requires the activation of caspases, A431 cells were exposed to 
caspase inhibitors including a pan caspase inhibitor (Z-VAD), and caspase-3, -8, and -9 inhibitors (Z-DEVD, Z-IETD, and Z-LEHD, respectively) for $30 \mathrm{~min}$ before treatment with $500 \mu \mathrm{g} / \mathrm{mL}$ GBT. As shown in Figure 3D, all caspase inhibitors inhibited the anti-proliferative effects of GBT, particularly Z-VAD and Z-DEVD. Taken together, these results suggest that GBT-induced apoptosis in A431 cells requires the activation of caspases.

\section{GBT regulates the phosphorylation of cell proliferation-} related proteins including MAPK cascades and p53 in A431 cells

To investigate the relationship between the activation of MAPKs and the inhibition of cancer cell proliferation, we analyzed the levels of total and phosphorylated
MAPKs by Western blotting after GBT treatment. The levels of p-p38 and p-ERK were upregulated after 15 min treatment with $500 \mu \mathrm{g} / \mathrm{mL}$ GBT, and the activation was sustained for $24 \mathrm{~h}$ (Figure $5 \mathrm{~A}$ ). In contrast, the phosphorylation of JNK was upregulated transiently by 30 min exposure to GBT, but then dropped sharply by $24 \mathrm{~h}$. The levels of p53 phosphorylated on Ser15 (p53$\operatorname{Ser}^{15} \mathrm{P}$ ) were increased after $6 \mathrm{~h}$ treatment with GBT, and expression remained elevated until $24 \mathrm{~h}$. To further investigate the regulation of these signaling pathway by GBT, A431 cells were pretreated with MAPK inhibitors, including PD98059 (which inhibits ERK1/2), SB203580 (p38), and SP600125 (JNK) for $30 \mathrm{~min}$, followed by treatment with $500 \mu \mathrm{g} / \mathrm{mL}$ GBT for $48 \mathrm{~h}$ (Figure 5B). Each inhibitor abrogated the anti-proliferative effects of






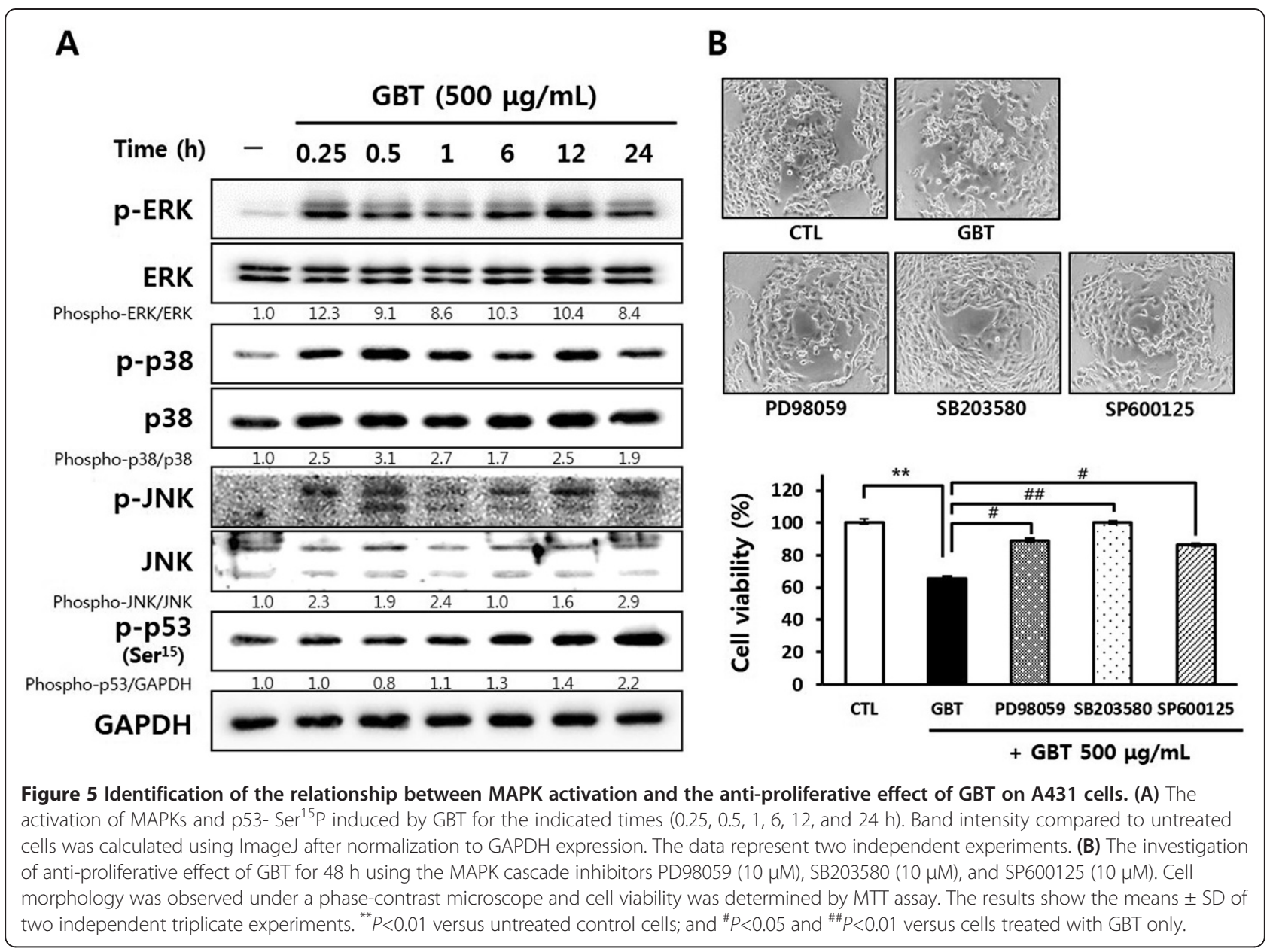

GBT in A431 cells significantly. In particular, SB203580 reduced cell death by $30 \%$ compared to GBT alone. Taken together, these data suggest that GBT has anti-proliferative effects on A431 cells by modulating MAPK signaling pathways, resulting in the induction of apoptosis.

\section{GBT administration inhibits tumorigenic growth of A431 cells in vivo}

To confirm these observations, we assessed the inhibitory effects of GBT on tumor growth in athymic nude mice injected with A431 cells. Mice harboring xenograft tumors were treated orally with either vehicle (control) or $600 \mathrm{mg} / \mathrm{Kg} /$ day GBT. Importantly, arrest in the growth of xenografts treated with GBT was observed after 7 days of treatment, followed by a significant reduction in tumor size at the end of experiment on day 15. In addition, GBT did not cause any adverse side effects such as loss of body weight or skin ulcers (Figure 6A, B). Treatment with GBT resulted a $52 \%$ inhibition of tumor growth compared to vehicle (Figure $6 \mathrm{C}, \mathrm{D}$ ). There were no significant differences in serological parameters among the three groups (Table 3). The ratios of GOT/GPT and BUN/CRE were not significantly changed in the GBT- treated group compared to control. In addition, the lactate dehydrogenase (LDH) ratio was decreased by $\sim 23 \%$ by GBT treatment, suggesting that GBT did not cause hepatic or renal damage. The hematological parameters of the GBT-treated group were similar to the vehicle group (Table 4). The number of red blood cells (RBCs) and hemoglobin levels, an indicator of RBC count and anemia, was unchanged by GBT. White blood cell (WBC) counts and other parameters were also within the normal ranges. Therefore, these results provide strong evidence for the anti-cancer effects of GBT in vivo.

\section{Discussion}

Traditional medicine in Asian countries commonly combines herbs to create multi-herbal formulas for treating target diseases, and the use of these formulas has been verified scientifically as complementary or alternative cancer therapies [16]. Especially, advanced diseases including cancer require the multi-targeting treatment in cellular signaling pathways, herbal formulas may achieve better therapeutic efficacy according to the synergy than that of a single herb [17]. However, multi-herbal formulas must be pre-clinically evaluated to accurately compare 


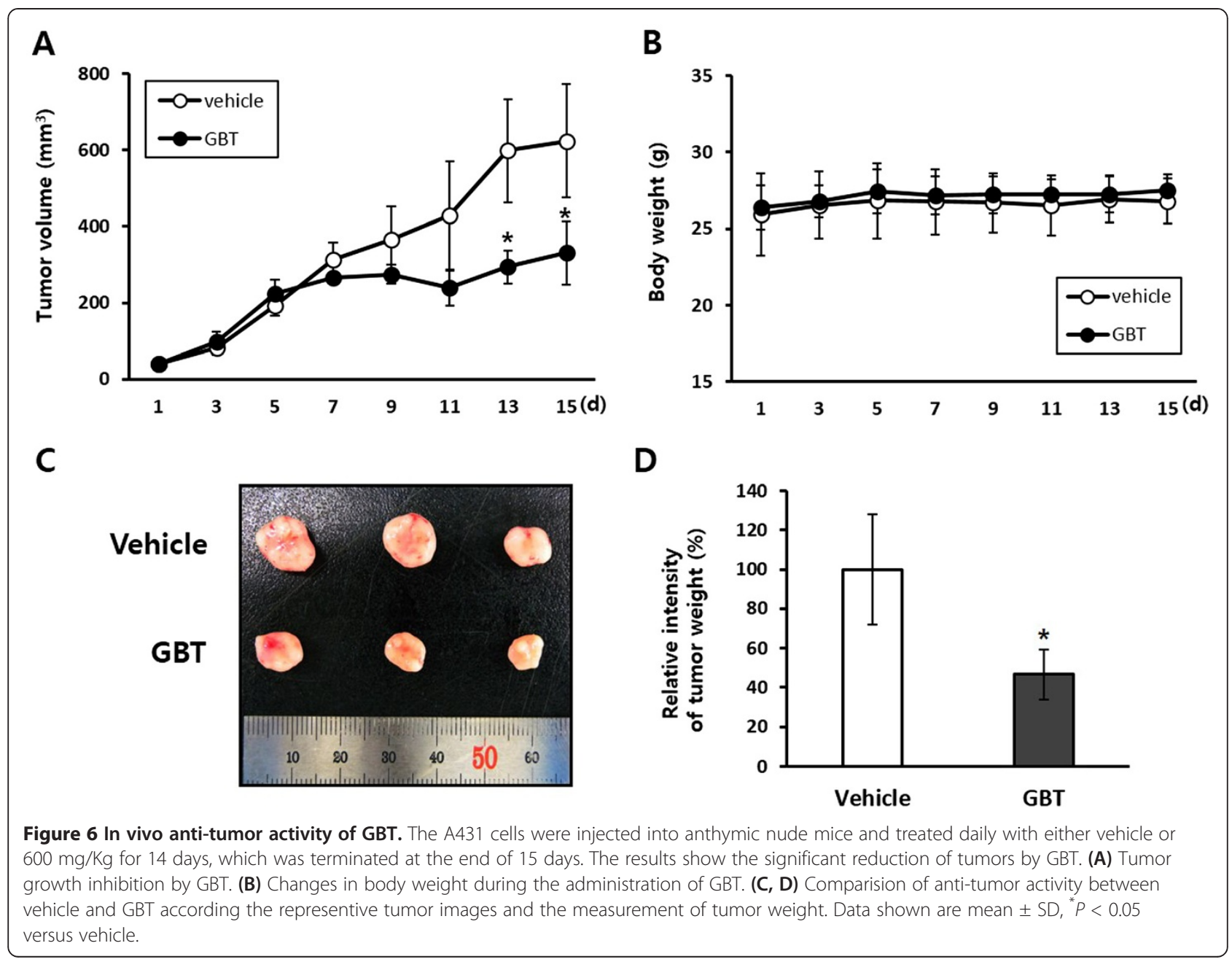

traditional herbal medicines with modern therapeutics [18]. In the present study, 14 standards in 12 constituent herbals from GBT were identified in the GBT samples. A previous study has reported that decursin, decursinol, and decursinol angelate from $A$. gigas Nakai indicate the anti-cancer effects in colon and breast carcinoma cells via inhibition of proliferation and induction of apoptosis $[19,20]$. Ginsenoside Rg1 and Rb1 from P. ginseng have anti-proliferative effect in colon cancer via cell cycle arrest and apoptosis induction [21]. A recent study has reported that vanilylacetone from $Z$. offcinale Rosc. has anti-carcinogenic properties against colon cancer, and 6- gingerol has apoptotic effect against breast and prostate carcinoma cells via modulation of STAT3 and MAPK signaling pathway $[22,23]$. In addition, formononetin from A. membranaceus induces apoptosis in prostate cancer cells via enhancing the $\mathrm{Bax} / \mathrm{Bcl}-2$ ratios and regulating the p38 phosphorylation [24]. Other reports have demonstrated that sesquiterpenoids from $A$. ovate, such as atractylenolide I, II, and III, exists anti-tumor effect in lung carcinoma cells via caspase-dependent apoptosis pathway $[25,26]$. These reports suggest that the anticancer effect of GBT might be related to these active components. In this study, GBT induced apoptosis in

Table 3 Chemical analysis of serums obtained from mice administrated with $600 \mathrm{mg} / \mathrm{Kg}$ of GBT

\begin{tabular}{ccccccc}
\hline Treat. & GOT (IU/L) & GPT (IU/L) & ALP (IU/L) & LDH (IU/L) & UREA (mg/dL) & CRE (mg/dL) \\
\hline Vehicle & $54.0 \pm 3.5$ & $28 \pm 0.0$ & $48.0 \pm 22.3$ & $420.0 \pm 65.8$ & $27.9 \pm 7.6$ & $1.0 \pm 0.0$ \\
GBT & $41.3 \pm 4.6$ & $26 \pm 0.0$ & $30.7 \pm 21.9$ & $319.3 \pm 94.7$ & $27.5 \pm 0.2$ & $1.0 \pm 0.0$
\end{tabular}

Each group of mice $(n=5)$ was treated orally with $600 \mathrm{mg} / \mathrm{Kg} \mathrm{GBT}$ for 14 days. The levels of GOT, GPT, ALP, LDH, BUN, and CRE were analyzed to confirm the cytotoxic effects of GBT. Data are expressed as mean \pm S.D.

GOT, glutamic oxaloacetic transaminase; GBT, glutamic puruvic transaminase; ALP, alkaline phosphatase; LDH, lactate dehydrogenase; BUN, blood urea nitrogen; $\mathrm{CRE}$, creatinine. 
Table 4 Hematological analysis of bloods obtained from mice administrated with $600 \mathrm{mg} / \mathrm{Kg}$ of GBT

\begin{tabular}{|c|c|c|}
\hline Parameters & Vehicle & GBT \\
\hline$\overline{\text { WBCP }\left(\times 10^{3} \text { cells } / \mu \mathrm{L}\right)}$ & $4.48 \pm 1.22$ & $4.02 \pm 1.19$ \\
\hline WBCB $\left(\times 10^{3}\right.$ cells $\left./ \mu \mathrm{L}\right)$ & $4.53 \pm 1.15$ & $4.10 \pm 1.24$ \\
\hline $\mathrm{RBC}\left(\times 10^{6}\right.$ cells $\left./ \mu \mathrm{L}\right)$ & $8.74 \pm 0.26$ & $8.99 \pm 0.48$ \\
\hline Means HGB (g/dL) & $13.8 \pm 0.06$ & $14.0 \pm 1.11$ \\
\hline $\mathrm{HCT}(\%)$ & $47.8 \pm 1.40$ & $49.1 \pm 1.81$ \\
\hline MCV (fL) & $54.7 \pm 0.40$ & $54.6 \pm 0.86$ \\
\hline $\mathrm{MCH}(\mathrm{pg})$ & $15.8 \pm 0.40$ & $15.6 \pm 0.40$ \\
\hline $\mathrm{MCHC}(\mathrm{g} / \mathrm{dL})$ & $28.8 \pm 0.74$ & $28.6 \pm 1.15$ \\
\hline PLT (x10 cells/mL) & $11.4 \pm 7.35$ & $11.3 \pm 11.17$ \\
\hline$\%$ NEUT & $21.7 \pm 1.63$ & $26.0 \pm 4.76$ \\
\hline$\%$ LYM & $71.7 \pm 1.81$ & $67.2 \pm 5.78$ \\
\hline$\%$ MONO & $1.40 \pm 0.35$ & $1.30 \pm 0.44$ \\
\hline
\end{tabular}

Each group of mice $(n=5)$ was treated orally with $600 \mathrm{mg} / \mathrm{Kg}$ GBT for 14 days, and leucocytes were analyzed. Data are expressed as mean \pm S.D.

WBCP, white blood cell count peroxidase method; $W B C B$, white blood cell count basophile method; RBC, red blood cell count; $\mathrm{HGB}$, hemoglobin; $\mathrm{HCT}$, hematocrit; $\mathrm{MCV}$, mean corpuscular volume; $\mathrm{MCH}$, mean corpuscular hemoglobin; MCHC, mean corpuscular hemoglobin concentration; PLT, platelet; NEUT, neutrophil; LYM, lymphocyte; MONO, monocyte.

A431 human squamous carcinoma cells by inhibiting cancer cell proliferation without affecting the viability of $\mathrm{HaCaT}$ keratinocytes or mouse primary hepatocytes. Based on these preliminary observations, we assessed the molecular mechanism of the anti-cancer effects of GBT on A431 cells. GBT increased the formation of fragmented DNA ladders as well as other apoptotic features such as chromatin condensation. In Western blot analysis, GBT affected the expression of pro- and anti-apoptotic proteins, espectially, GBT enhanced the activation of caspases in A431 cells. Caspases are a family of cysteine proteases that, when inactive, exist in proenzyme form. Upon the induction of apoptosis, they become activation via a self-amplifying cascade [27]. The activation of initiator caspases such as caspases-8, -9 , and -10 by proapoptotic signals leads to downstream activation of the effector caspases-3, -6 , and -7 . The caspase cascades are divided into two major pathways: an extrinsic pathway initiated by ligand-mediated activation of cell surface death receptors, and an intrinsic pathway activated by intracellular signals from the mitochondria [28]. In the present study, the activation of caspase- 8 and -3 and the cleavage of PARP correlated precisely with the DNA fragmented ladder after treatment with GBT, whereas the levels of procaspase- 9 decreased slightly, without the appearance of the cleaved form. The Bcl-2 family of proteins, including Bid, Bcl-2, and Bax, regulate the activation of caspase-9 [29]. In our study, GBT-stimulated active caspase- 8 did not increase the levels of truncated Bid, although total Bid levels were reduced. Our data suggest that the activation of caspase- 8 by GBT results in the direct activation of caspase-3, which is typical of the extrinsic apoptotic pathway, suggesting that GBT activates extrinsic apoptosis to have anti-cancer effects on A431 cells. We also found that GBT stimulated the phosphorylation of MAPKs and p53, signaling pathways that are required for cell growth and tumorigenesis. MAPK cascades including ERK, p38, and JNK, regulate cellular processes including proliferation, differentiation, and apoptosis [30]. Especially, Pharmacological modulation of MAPK singals has been confirmed in previous studies to influence the apoptotic response to anti-tumor agents. For example, ERK activation by treatment with cisplatin plays a key role in mediating cisplatin-induced apoptosis of HeLa human cervical carcinoma cells, which induces caspase activation [31]. Another example is represent that the role of MAPK and p53 pathways in cancer cells is associated with anti-cancer effect of chemotherapeutic agents such as vinblastine, doxorubicin and etoposide [32]. In the present study, we identified that GBT treatment activated the ERK, p38, and JNK signals, which retained during apoptosis of A431 cells. In addition, inhibition of MAPK signaling by the specific inhibitors (PD98059, ERK inhibitor; SB203580, p38 inhibitor; SP600125, JNK inhibitor) protected cells from the cytotoxic effects of GBT, suggesting that activation of MAPK cascades play a opposite role in A431 cell proliferation. MAPKs are activated upon exposure to stress, leading to the phosphorylation and activation of $\mathrm{p} 53$. The activation of MAPKs can activate p53 to phosphorylation form at various serine residues, resulting in p53-mediated cellular responses such as DNA repair, cell cycle arrest, and the induction of apoptosis [33]. The phosphorylation of p53 at serine $15\left(\mathrm{p} 53-\operatorname{Ser}^{15} \mathrm{P}\right)$ by $\mathrm{p} 38$ or ERK results in the induction of apoptosis in cancer cells [34,35]. In contrast, activated JNK plays a direct role in the phosphorylation of p53 at serine 20, leading to the activation and stabilization of p53. In cell cycle progression relevant to cell proliferation, furthermore, the activation of p53 causes cell cycle arrest in the G1 phase, which mediated by $\mathrm{p} 21$ and $\mathrm{p} 27$, inhibitors of cyclin/CDK complexes $[36,37]$. In present study, the activation of ERK, p38, and JNK by GBT corresponded with increase of $\mathrm{p} 53-\mathrm{Ser}^{15} \mathrm{P}$ expression in A431 cells, which caused the up-regulation of p21 and p27 expressions during GBT-induced apoptosis. Taken together, these results strongly suggest that activation of MAPK cascades by GBT induces phosphorylation of $\mathrm{p} 53$, which results in induction of apoptosis for A431 cells.

Based on results demonstrated in A431 cells in vitro, we performed xenograft assay in athymic nude mice. In the evaluation of inhibitory effect of GBT against tumor growth after 15 days of daily oral administration, GBT significantly suppresses tumor growth of subcutaneously injected A431 cells without side effects such as body 
weight loss, organ abnormalities, and hematological/serological parameter changes. Therefore, GBT has a significant anti-tumorigenic effect in vivo.

\section{Conclusions}

This study assessd the efficacy of GBT anti-cancer effect in vitro and vivo. Our results strongly demonstrated that GBT induced apoptosis by regulating the activity of MAPK cascades and p53 in A431 cells. Further, oral administration of GBT obviously inhibited in vivo tumor cell growth of A431 cells without causing systemic toxicity. Resultingly, we suggest that GBT has potential as a herbal medicine for controlling malignant tumor growth.

\section{Competing interests}

The authors declare that they have no competing interests.

\section{Authors' contributions}

$\mathrm{NHY}$ conceived of the study, carried out overall in vitro and in vivo experiments, and drafted manuscript. AYK participated in animal experiments and discussed results. CL analyzed the constituents of GBT. JYM and WKC participated in the design and coordination of study. All authors read and approved the final manuscript.

\section{Acknowledgments}

This work was supported by a grant (K14050) awarded to the Korean Institute of Oriental Medicine by the Ministry of Education, Science and Technology (MEST), Korea. Further, we thank Ju Hye Lee for helpful experiment in this study.

Received: 22 April 2014 Accepted: 16 September 2014 Published: 21 September 2014

\section{References}

1. Diepgen $\mathrm{TL}$, Mahler $\mathrm{V}$ : The epidemiology of skin cancer. $\mathrm{Br} J$ Dermatol 2002, 146(Suppl 61):1-6.

2. Weinstein MC, Brodell RT, Bordeaux J, Honda K: The art and science of surgical margins for the dermatopathologist. Am J Dermatopathol 2012, 34(7):737-745

3. Sauter ER, Herlyn M, Liu SC, Litwin S, Ridge JA: Prolonged response to antisense cyclin D1 in a human squamous cancer xenograft model. Clin Cancer Res 2000, 6(2):654-660.

4. Trakatelli M, Ulrich C, del Marmol V, Euvrard S, Stockfleth E, Abeni D: Epidemiology of nonmelanoma skin cancer (NMSC) in Europe: accurate and comparable data are needed for effective public health monitoring and interventions. Br J Dermatol 2007, 156(Suppl 3):1-7.

5. Tohda C, Ichimura M, Bai Y, Tanaka K, Zhu S, Komatsu K: Inhibitory effects of Eleutherococcus senticosus extracts on amyloid beta(25-35)-induced neuritic atrophy and synaptic loss. J Pharmacol Sci 2008, 107(3):329-339.

6. Busta I, Xei HS, Kim MS: The use of Gui-Pi-Tang in small animals with immune-mediated blood disorders. J Vet Clin 2009, 26:181-184.

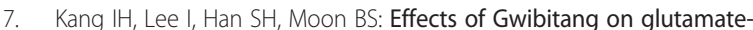
induced apoptosis in C6 glial cells. J Korean Orient Med 2001, 22:45-57.

8. Kim HJ, Choi JH, Lim SW: The defensive effect of Keuibi-tang on the gastric mucous membrane of mouse injured by stress and ethanol. J Orient Med 2003, 24:155-168.

9. Ikeda A, Higashio S, Ushiroyama T: Experience with administration of kamikihito with chemotherapy and palliative care in patients with gynecologic cancer. Recent Prog Kampo Med Obstet Gynecol 2003, 20:152-155

10. Kundoor $V$, Zhang $X$, Bommareddy A, Khalifa S, Fahmy H, Dwivedi C: Chemopreventive effects of sarcotriol on ultraviolet B-induced skin tumor development in SKH-1 hairless mice. Marine Drugs 2007. 5(4):197-207.

11. Sarfaraz S, Adhami VM, Syed DN, Afaq F, Mukhtar H: Cannabinoids for cancer treatment: progress and promise. Cancer Res 2008, 68(2):339-342.
12. Hamamura $\mathrm{K}$, Goldring MB, Yokota $\mathrm{H}$ : Involvement of p38 MAPK in regulation of MMP13 mRNA in chondrocytes in response to surviving stress to endoplasmic reticulum. Arch Oral Biol 2009, 54(3):279-286.

13. Fan $M$, Chambers TC: Role of mitogen-activated protein kinases in the response of tumor cells to chemotherapy. Drug Resist Updat 2001, 5:253-267.

14. Dent P, Grant S: Pharmacologic interruption of the mitogen-activated extracellular-regulated kinase/mitogen-activated protein kinase signal transduction pathway: potential role in promoting cytotoxic drug action. Clin Cancer Res 2001, 7:775-783.

15. Yim NH, Lee JH, Cho WK, Yang MC, Kwak DH, Ma JY: Decursin and decursinol angelate from Angelica gigas Nakai induce apoptosis via induction of TRAIL expression on cervical cancer cells. Eur J Integr Med 2011, 3:299-307.

16. Son JY, Choi HY, Kim JD: Anticancer and related immunomodulatory effects of Kwibi-tang on non-small cell lung carcinoma, NCl-H520, Xenograft mice. Korean J Interal Med 2012, 33:387-404.

17. Corson TW, Crews GM: Molecular understanding and modern application of traditional medicines: triumphs and trials. Cell 2007, 130:769-774.

18. Lee EO, Lee HJ, Hwang HS, Ahn KS, Chae C, Kang KS, Lu J, Kim SH: Potent inhibition of Lewis lung cancer growth by heyneanol A from the roots of Vitis amurensis through apoptotic and anti-angiogenic activities. Carcinogenesis 2006, 27(10):2059-2069.

19. Son SH, Park KK, Park SK, Kim YC, Kim YS, Lee SK, Cung WY: Decursin and decursinol from Angelica gigas inhibit the lung metastasis of murin colon carcinoma. Phytother Res 2011, 25(7):959-964.

20. Jiang C, Guo J, Wang Z, Xiao B, Lee HJ, Lee EO, Kim SH, Lu J: Decursin and decursinol angelate inhibit estrogen-stimulated and estrogenindependent growth and survival of breast cancer cells. Breast Cancer Res 2007, 9(6):R77

21. Wang CZ, Xie JT, Zhang B, Ni M, Fishbein A, Aung HH, Mehendale SR, Du W, He TC, Yuan CS: Chemopreventive effects of Panax notoginseng and its major constituents on SW480 human colorectal cancer cells. Int J Oncol 2007, 31(5):1149-1156.

22. Vinothkumar R, Vinothkumar R, Sudha M, Nalini N: Chemopreventive effect of zingerone against colon carcinogenesis induced by 1,2dimethylhydrazine in rats. Eur J Cancer Prev 2014, 23(5):361-371.

23. Kim SM, Kim C, Bae H, Lee JH, Baek SH, NAm D, Cung WS, Shim BS, Lee SG, Kim SH, Sethi G, Ahn KS: 6-shogaol exerts anti-proliferative and proapoptotic effects through the modulation of STAT3 and MAPKs signaling pathways. Mol Carcinog 2014. doi:10.1002/mc 22184.

24. Zhang $X, B i L$, Ye $Y$, Chen J: Formononetin induces apoptosis in PC-3 prostate cancer cells through enhancing the $\mathrm{Bax} / \mathrm{Bcl}-2$ ratios and regulating the p38/Akt pathway. Nutr Cancer 2014, 66(4):656-661.

25. Liu H, Zhu Y, Zhang T, Zhao Z, Zhao Y, Cheng P, Li H, Gao H, Su X: Anti-tumor effects of atractylenolide I isolated from Atractylodes macrocephala in human lung carcinoma cell lines. Molecules 2013, 18(11):13357-13368.

26. Kang TH, Bang JY, Kim MH, Kang IC, Kim HM, Jeong HJ: Atractylenolide III, a sesquiterpenoid, induces apoptosis in human lung carcinoma A549 cells via mitochondria-mediated death pathway. Food Chem Toxicol 2011, 49(2):514-519.

27. Saraste A, Pulkki K: Morphologic and biochemical hallmarks of apoptosis. Cardiovasc Res 2000, 45(3):528-537.

28. Sheikh MS, Huang Y: Death receptors as targets of cancer therapeutics. Curr Cancer Drug Targets 2004, 4(1):97-104.

29. Reyes-Zurita FJ, Rufino-Palomares EE, Medina PP, Leticia Garcia-Salguero E, Peragon J, Cascante M, Lupianez JA: Antitumour activity on extrinsic apoptotic targets of the triterpenoid maslinic acid in p53-deficient Caco-2 adenocarcinoma cells. Biochimie 2013, 95(11):2157-2167.

30. Murphy LO, Blenis J: MAPK signal specificity: the right place at the right time. Trends Biochem Sci 2006, 31(5):268-275.

31. Wang $X$, Martindale $J$, Holbrook NJ: Requirement for ERJK activation in cisplatin-induced apoptosis. J Biol Chem 2000, 275:39435-39443.

32. Cheryl BF, Christopher SL, Lihua D, Mary EG, Toria H, Dominika S, Kaushal GP, Timothy CC: The JNK, ERK and p53 pathways play distinct roles in apoptosis mediated by the antitumor agents vinblastine, doxorubicin, and etoposide. Biol Pharmacol 2003, 66:459-469.

33. Jiang C, Lee HJ, Li GX, Guo J, Malewicz B, Zhao Y, Lee EO, Lee HJ, Lee JH, Kim MS, Lu J: Potent antiandrogen and androgen receptor activities of an Angelica gigas-containing herbal formulation: identification of 
decursin as a novel and active compound with implications for prevention and treatment of prostate cancer. Cancer Res 2006, 66(1):453-463.

34. Kim SJ, Hwang SG, Shin DY, Kang SS, Chun JS: p38 kinase regulates nitric oxide-induced apoptosis of articular chondrocytes by accumulating p53 via NFkappa B-dependent transcription and stabilization by serine 15 phosphorylation. J Biol Chem 2002, 277(36):33501-33508.

35. She QB, Bode AM, Ma WY, Chen NY, Dong Z: Resveratrol-induced activation of p53 and apoptosis is mediated by extracellular-signalregulated protein kinases and p38 kinase. Cancer Res 2001, 61(4):1604-1610.

36. Koljonen V: Merkel cell carcinoma. World J Surg Oncol 2006, 4:7.

37. Wang $X$, Gorospe $M$, Huang $Y$, Holbrook NJ: p27Kip1 overexpression causes apoptotic death of mammalian cells. Oncogene 1997, 15(24):2991-2997.

doi:10.1186/1472-6882-14-344

Cite this article as: Yim et al:: Guibitang, a traditional herbal medicine, induces apoptotic death in A431 cells by regulating the activities of mitogen-activated protein kinases. BMC Complementary and Alternative Medicine 2014 14:344.

\section{Submit your next manuscript to BioMed Central and take full advantage of:}

- Convenient online submission

- Thorough peer review

- No space constraints or color figure charges

- Immediate publication on acceptance

- Inclusion in PubMed, CAS, Scopus and Google Scholar

- Research which is freely available for redistribution 